\title{
El profesor estratégico como favorecedor del clima de aula
}

\author{
Miguel Á. Carbonero, Luis J. Martín-Antón y Natalia Reoyo \\ Universidad de Valladolid (España)
}

El presente trabajo pretende contribuir a la tesis de que un profesor estratégico es una forma distinta y eficaz de contribuir a la mejora del clima del aula. Para ello se planteó una situación experimental en la que se entrenó a un grupo de profesores en habilidades docentes motivadoras a través de la utilización de estrategias de enseñanza basadas en la instrucción, evaluación, habilidades sociales y motivación. Se llevó a cabo con profesores de $1^{\circ}$ y $3^{\circ}$ de la ESO de varios centros, que impartían clase a 264 alumnos de $1^{\circ}$ y 253 de $3^{\circ}$ de la ESO. El instrumento utilizado para medir el clima de aula fue el Cuestionario de clima social en la clase (CES), tomando seis de las variables propuestas: Implicación, Ayuda, Tareas, Competitividad, Organización, e Innovación. Los resultados nos indican una eficacia del programa en tercero de ESO en todas las variables analizadas del Clima del aula, frente a primero, que únicamente mejoran en Competitividad e Innovación. Respecto al sexo, no se producen diferencias en Primero. En tercero hay una mayor eficacia del programa en las mujeres en Autoeficacia para el rendimiento, justo lo contrario que en Implicación, Ayuda y Organización. Respecto al curso, se produce una mejora en Tercero de ESO en las variables Implicación, Tareas y Organización que no ocurre en Primero de ESO.

Palabras clave: Profesor estratégico, clima de aula, Educación Secundaria.

The strategic teacher as becoming of classroom environment. The aim of this paper is contributing to the proposition that a strategic teacher is a different and effective way to contribute to improving the classroom climate. An experimental situation was proposed to train a group of teachers in motivating teaching skills through learning strategies based on instruction, assessment, social skills and motivation. It was carried out by secondary teachers from several schools who taught classes to 264 high school students in first grade and 253 in third grade. The instrument used to measure the classroom climate was the Classroom Environment Scale (CES), taking six of the suggested variables: Involvement, Teacher support, Task orientation, Competition, Order and organization and Innovation. The results point out efficacy of the program in third grade in all the variables, compared to the results of first grade that only improve in Competition and Innovation. Regarding gender, no differences were found in first grade. However, there are more efficacy of the program in women of third grade in Self-efficacy of performance, just the opposite that in Involvement, Teacher Support and Order and Organization. With regard to grades, an improvement is produced in third grade in the variables of Involvement, Task orientation and Order and organization that doesn't occur in first grade.

Key words: Strategic teacher School climate, secondary student.

Correspondencia: Miguel Ángel Carbonero Martín. Facultad de Educación y Trabajo Social. Campus Miguel Delibes. Paseo Belén nº1. 47011. Valladolid. E-mail: carboner@psi.uva.es 
Son pocos los estudios que relacionan las habilidades docentes del profesorado con el rendimiento de los estudiantes. Hay estudios que demuestran como la calidad, el estilo de enseñanza, las estrategias que emplean... e incluso sus propias expectativas, va a influir en el incremento de la motivación y el rendimiento académico de sus alumnos (Woolfolk, 2006).

A pesar de ello, actualmente no se ha superado el modelo tradicional de educación, lo que podría explicar, (la falta de adaptación del profesorado) la dificultad de un gran número de profesores a la hora de adaptarse al modelo actual centrado en el alumno, en la adquisición de competencias, problemas de comportamiento...; y la insuficiente motivación y bajo éxito académico del alumnado. Dejándose así presente de forma notoria, la gran necesidad de una formación permanente con un mayor rigor pedagógico y propuestas metodológicas más específicas, que permitan al profesorado adquirir habilidades que realmente le permitan enfrentarse a los nuevos desafíos educativos actuales (o nueva situación educativa actual) (Benito, 2009). Aunque al alumno se le ha otorgado el papel principal dentro del proceso de enseñanza-aprendizaje, su éxito académico y motivación no sólo depende de él, sino de también de otras variables, entre ellas el profesorado, pilar principal de nuestra investigación (Valle, González-Cabanach y Rodríguez, 2006).

Los efectos de la enseñanza en el alumnado van a ser decisivos en las primeras etapas educativas, pero también en años posteriores, encontrarse con profesorado que ponga en juego habilidades docentes eficaces se puede convertir en una forma efectiva de recuperar a estudiantes con baja motivación y rendimiento académico.

La falta de motivación en las aulas se ha convertido en uno de los problemas centrales de la educación en España (Valle et al., 2006) y son numerosas las investigaciones que ya desde hace un tiempo muestran como la motivación influye significativamente en el aprendizaje del alumnado (Alonso-Tapia, 2005). Se ha observado como alumnos con una media o escasa motivación obtienen resultados más bajos en rendimiento académico (Lozano, 2005) y en el uso de estrategias de aprendizaje. Por tanto las carencias en habilidades de enseñanza adecuadas van a contribuir a la desmotivación del alumnado, condicionante principal de su poca implicación en las tareas escolares, el escaso e inadecuado uso de estrategias de aprendizaje, y del bajo rendimiento académico y desfavorable clima escolar (Benito, 2009; Carrasco, 2010; Fernández, 2008).

Debido al destacado papel activo que posee el estudiante en el proceso de enseñanza-aprendizaje, mayoritariamente los estudios dirigidos hacia este constructo se han basado en él, considerándolo como una de las variables principales que propician esta motivación o falta de motivación. Pero si la intervención se dirige hacia el 
profesorado cómo va a condicionar esta formación a la motivación, el uso de estrategias de aprendizaje y el clima escolar de la clase.

La filosofía de logro de una verdadera educación de calidad debería llevar al profesorado a una reflexión constante sobre los procesos de enseñanza y aprendizaje, asegurando así la aplicación de unas habilidades eficaces para la formación de nuestros alumnos.

La escuela como institución y el profesor como agente socializador se enfrentan al reto de abrir las puertas del siglo XXI, introduciendo cambios en su organización y en su quehacer en aras a que estos se lleven a cabo no sólo en el discurso sino en el accionar cotidiano del profesor. Es cierto que "los alumnos pueden y deben aprender por si mismos, pero también lo es que el aprendizaje puede hacerse con más rapidez y eficacia si se les ayuda" (Román, 2006).

Cuanto más alta sea la relación entre el estilo de enseñanza del profesor y el estilo de aprendizaje del alumno, más altos serán los logros de los estudiantes (Hervás, 2005). Por ello, es imprescindible que el profesorado, conozca que: a) los diferentes modos de enseñar implica diferentes modos de aprender, b) hay diferentes tipos de enseñanza para diferentes tipos de aprendizaje, c) la actividad del alumno guarda una estricta relación con la enseñanza que ha recibido, y que d) el trabajo del docente se refleja en el aprendizaje y motivación del estudiante.

Son numerosas las investigaciones que dentro del campo de la educación hablan de la importancia del docente a la hora de lograr el éxito en el aprendizaje de los alumnos (Gage, 1972; Brophy y Good, 1996), éxito que exige un docente con amplios conocimientos del qué enseñar, cómo hacerlo, y que posea o sea capaz de adquirir competencias (Angulo, 1999; ANECA, 2004; Fernandez, 2008; García-Llamas, 1999; Marchesi, 2007; Perrenoud, 2004; Zabalza, 2003) y estilos de enseñanza efectivos.

Lo importante es la utilización de diferentes y variados estilos de enseñanza (Beltrán, 1987; Bennett, 1979; Flanders, 1984; Martínez-Geijo, 2007; Gregorc, 1985) para atender a la diversidad de los alumnos, incluyendo en las programaciones algunas variables relacionadas con los estilos y el aprendizaje. Es fundamental para el profesor conocer qué es lo que está haciendo, por qué y creer en ello (Gregory, 1985), siendo así, capaces de tener unos propósitos y unos objetivos claros, utilizando las estrategias y técnicas instructivas idóneas para motivar y apoyar a los estudiantes de forma adecuada, controlando la enseñanza y evaluando la importancia del aprendizaje (Hervás, 2005).

Como indican Sternberg (1990) y Prieto y Hervás (1995), el estilo de enseñanza es el que gobierna la realidad de la clase. Los educadores necesitan conocer y considerar sus propios estilos para entender cómo influyen sus percepciones e interacciones con los demás y necesitan, a su vez, conocer los estilos de sus alumnos para poder desarrollarlos y así favorecer el aprendizaje. Conocidos ambos, deberán 
plantear las tareas de maneras diferentes para que beneficien a los que también poseen diferentes estilos intelectuales (Hervás, 2005).

La actividad docente no debe basarse exclusivamente en un solo estilo de enseñanza, sino que la acción educativa debe ser flexible y dinámica, atendiendo a la diversidad del alumnado y a los estilos de aprendizaje, utilizando una serie de pautas de actuación docente que contribuyan a mejorar el clima de aula.

El objetivo fundamental de esta investigación es profundizar en la comprensión de cómo una adecuada formación en habilidades docentes es un factor determinante para la mejora del clima de aula.

\section{Objetivos}

Conocer la eficacia del programa en la mejora del clima de aula en el $1^{\circ}$ y $3^{\circ}$ curso de ESO.

Analizar si existe alguna interacción en la eficacia del programa en la variable clima de aula en función de la variable sexo y curso.

\section{METODO}

\section{Participantes}

Se han seleccionado al alumnado del primer y tercer curso de Educación Secundaria Obligatoria de nueve centros educativos, tanto públicos como concertados, de la ciudad de Valladolid. Estos centros fueron asignados aleatoriamente a dos grupos:

Grupo experimental: Se realizó un entrenamiento sobre la importancia de tener en cuenta ciertas estrategias docentes que, al menos teóricamente, mejoran el clima escolar del aula.

Grupo control: no se realizó intervención alguna con este profesorado.

La muestra de Primero de ESO estaba formada por 264 sujetos, con una edad media de 12.7 años. En cuanto al sexo, el 51.5\% eran mujeres y el $48.5 \%$ varones. Respecto a Tercero de ESO, han participado 253 alumnos y alumnas con una edad media de 14.9 años, y en los que el $54.5 \%$ eran mujeres y 45.5 fueron varones. La distribución entre el grupo experimental y control tal es prácticamente proporcional en ambos cursos, como se muestra en la tabla 1.

\begin{tabular}{clcc}
\multicolumn{3}{c}{ Tabla 1. Distribución de la Muestra por Curso y Condición Experimental } \\
\hline & & Frecuencia & Porcentaje \\
\hline \multirow{2}{*}{$1^{\circ}$ ESO } & Experimental & 145 & 54.9 \\
& Control & 119 & 45.1 \\
& Total & 264 & 100.0 \\
\hline \multirow{2}{*}{$3^{\circ}$ ESO } & Experimental & 137 & 54.2 \\
& Control & 116 & 45.8 \\
& Total & 253 & 100.0 \\
\hline
\end{tabular}




\section{Variables}

Variable independiente: programa de entrenamiento a profesores en habilidades docentes motivadoras, con dos condiciones experimentales: (a) experimental, que ha seguido el programa; y (b) control, que no ha recibido información alguna.

Variable independiente 2: Curso: (a) Primero de ESO; y (b) Tercero de ESO Variable dependiente: el clima de aula.

\section{Instrumentos}

Cuestionario de clima social en la clase (CES): Elaborado por E.D.Trickett y R.H. Moos (1974); Adaptación española de R. Fernández-Ballesteros y B. Sierra (1988). Esta escala evalúa el clima social en clases de enseñanza media y superior, atendiendo a la medida y descripción de las relaciones profesor-alumno y a la estructura organizativa de la clase. Las subescalas que hemos considerado son:

Implicación. Mide el grado en que los alumnos muestran interés por las actividades de la clase y participan en los coloquios y como disfrutan del ambiente creado incorporando tareas complementarias.

Ayuda: Grado de ayuda, preocupación y amistad del profesor por los alumnos.

Tareas: Importancia que se da a la terminación de las tareas programadas. Énfasis que pone el profesor en el temario de la asignatura.

Competitividad: Grado de importancia que se da al esfuerzo por lograr una buena calificación y estima, así como a la dificultad para obtenerlas.

Organización: Importancia que se da al orden, organización y buenas maneras en la realización de las tareas escolares.

Innovación: Grado en que los alumnos contribuyen a planear las actividades escolares y la variedad y cambios que introduce el profesor con nuevas técnicas y estímulos a la creatividad del alumno.

\section{Procedimiento}

En primer lugar se contactó con los centros. Aleatoriamente se distribuyeron a grupo experimental y control (Carbonero, Martín-Antón y Feijoó, 2010). Posteriormente, se evaluó a los profesores en función del nivel motivador de sus habilidades docentes. A continuación, al profesorado del grupo experimental siguieron un entrenamiento en habilidades docentes motivadoras, consistente en:

Este proceso de formación consta de varias sesiones de entrenamiento, para el profesorado implicado, sobre los cuatro bloques del programa de "habilidades docentes motivadoras": (a) habilidades docentes de instrucción, (b) evaluación, (c) motivación y (d) habilidades sociales que consideramos oportunas que pongan en marcha en la clase al explicar los temas. 
Se inició la formación, procediendo a indicar a los profesores la selección de los temas comunes de cada materia sobre los que se van a implementar las Habilidades Docentes y la aplicación del cuestionario pre-test.

Las sesiones consisten en explicaciones de un ponente, dinámicas de grupo y trabajos independientes de aprendizaje y reflexión. En todas ellas se les facilita y presenta un material a aprender, en relación a todas las variables que en cada momento se van trabajando dentro del bloque de habilidades de instrucción, evaluación, habilidades sociales y motivación. Este material consiste en un dossier teórico-práctico cuyo contenido aborda las diferentes tipos de estrategias que serán aplicadas, con sus finalidades y procedimientos necesarios para la puesta en práctica en el aula.

Una vez completado todo el proceso instruccional, comenzaremos con la puesta en marcha por los profesores de cada materia de aquellas habilidades trabajadas con anterioridad. También se les proporcionó, en otra sesión formativa, una serie de criterios comunes de evaluación (a nivel conceptual / práctico / analítico, ya mencionados anteriormente), para la elaboración de las pruebas de contenidos sobre los temas y que serán aplicados inmediatamente después de su exposición.

Finalmente, se realizó una evaluación al alumnado asignado al grupo experimental y control sobre su percepción sobre el clima de aula.

Para el análisis de los datos se ha utilizado el paquete estadístico SPSS v.15, en donde, una vez comprobado el cumplimiento de los supuestos paramétricos, se han empleado pruebas de contraste de medias y análisis Multivariado de la varianza; y sus alternativas no paramétricas, según sea el caso.

\section{Diseño}

Se ha optado por un diseño cuasi-experimental, de postest con grupo experimental y control equivalente. Aunque la asignación de los centros al grupo experimental y control ha sido aleatoria, el alumnado es el que pertenece a cada centro y aula, por lo que se ha trabajo con grupos naturales.

\section{RESULTADOS}

En Primero de ESO nos encontramos con diferencias estadísticamente significativas en las variable Competitividad $(p<.001)$ e Innovación $(p<.01)$, todas superiores en el grupo experimental respecto al control (Tabla 2).

En cuanto a Tercero de ESO, nos encontramos con diferencias estadísticamente significativas en todas las variables correspondientes a la motivación: Implicación $(p<.01)$, Ayuda $(p<.001)$, Tareas $(p<.01)$, Competitividad $(p<.001)$, Organización $(p<.001)$ e Innovación $(p<.01)$, todas a favor del grupo experimental (Tabla 3). 
CARBONERO, MARTÍN-ANTÓN y REOYO. El profesor estratégico y clima escolar

Tabla 2. Diferencias en Clima de Aula en Primero de ESO

\begin{tabular}{|c|c|c|c|c|c|c|}
\hline & Grupo & $N$ & Media & D.T. & $U$ & Sig. \\
\hline \multirow{2}{*}{ Implicación } & Experimental & 143 & 4.24 & 2.470 & \multirow{2}{*}{7662.000} & \multirow{2}{*}{.163} \\
\hline & Control & 119 & 4.57 & 2.476 & & \\
\hline \multirow{2}{*}{ Ayuda } & Experimental & 144 & 6.32 & 1.769 & \multirow{2}{*}{7518.500} & \multirow{2}{*}{.083} \\
\hline & Control & 119 & 5.76 & 2.378 & & \\
\hline \multirow{2}{*}{ Tareas } & Experimental & 136 & 5.58 & 1.438 & \multirow{2}{*}{7414.000} & \multirow{2}{*}{.288} \\
\hline & Control & 118 & 5.71 & 1.845 & & \\
\hline \multirow{2}{*}{ Competitividad } & Experimental & 145 & 6.42 & 1.619 & \multirow{2}{*}{$6367.500 * * *$} & \multirow{2}{*}{.000} \\
\hline & Control & 119 & 5.75 & 1.421 & & \\
\hline \multirow{2}{*}{ Organización } & Experimental & 141 & 4.57 & 2.655 & \multirow{2}{*}{7731.500} & \multirow{2}{*}{.272} \\
\hline & Control & 119 & 4.82 & 2.577 & & \\
\hline \multirow{2}{*}{ Innovación } & Experimental & 143 & 4.75 & 1.651 & \multirow{2}{*}{$6764.500 * *$} & \multirow{2}{*}{.004} \\
\hline & Control & 119 & 4.13 & 1.741 & & \\
\hline
\end{tabular}

Tabla 3. Diferencias en Clima de Aula en Tercero de ESO

\begin{tabular}{|c|c|c|c|c|c|c|}
\hline & Grupo & $N$ & Media & D.T. & $U$ & Sig. \\
\hline \multirow{2}{*}{ Implicación } & Experimental & 136 & 4.79 & 3.111 & \multirow{2}{*}{$6347.000^{* *}$} & \multirow{2}{*}{.007} \\
\hline & Control & 116 & 3.68 & 2.569 & & \\
\hline \multirow{2}{*}{ Ayuda } & Experimental & 136 & 6.45 & 2.638 & \multirow{2}{*}{$5599.000 * * *$} & \multirow{2}{*}{.000} \\
\hline & Control & 116 & 5.16 & 2.569 & & \\
\hline \multirow{2}{*}{ Tareas } & Experimental & 135 & 5.93 & 1.817 & \multirow{2}{*}{$5027.500 * * *$} & \multirow{2}{*}{.000} \\
\hline & Control & 116 & 4.83 & 1.528 & & \\
\hline \multirow{2}{*}{ Competitividad } & Experimental & 136 & 6.26 & 1.679 & \multirow{2}{*}{$5978.500^{* *}$} & \multirow{2}{*}{.002} \\
\hline & Control & 113 & 5.56 & 1.620 & & \\
\hline \multirow{2}{*}{ Organización } & Experimental & 135 & 5.63 & 3.365 & \multirow{2}{*}{$5839.500 * * *$} & \multirow{2}{*}{.000} \\
\hline & Control & 116 & 4.18 & 2.727 & & \\
\hline \multirow{2}{*}{ Innovación } & Experimental & 134 & 4.78 & 1.696 & \multirow{2}{*}{$6260.000^{* *}$} & \multirow{2}{*}{.007} \\
\hline & Control & 116 & 4.16 & 1.923 & & \\
\hline
\end{tabular}

En cuanto a la interacción en función del seguimiento del programa y la variable Sexo. encontramos efectos diferenciales en las variables Implicación. Ayuda y Organización (Tabla 4) en Tercero de ESO. En cuando a la Implicación ( $p<.01)$. nos encontramos con una muy superior puntuación de los varones del grupo experimental respecto al control mientras que en las mujeres se producen puntuaciones similares en ambos grupos. En la variable Ayuda $(p<.05)$. nos encontramos con una mayor puntuación de los varones respecto a las mujeres del grupo experimental. Sin embargo. en el control ocurre todo lo contrario. La misma tendencia se produce en la variable Organización $(p<.01)$. En definitiva. podemos considerar que. en estas tres variables del Clima de Aula. el programa ha sido diferencialmente más eficaz en los varones respecto a las mujeres. Aún así, existen diferencias entre estadísticamente significativas a favor de las mujeres del grupo experimental frente a las del control en las variables Ayuda y Organización, sin embargo, en implicación no hay diferencias entre las puntuaciones de las mujeres de estos dos grupos.

Por último, en cuanto a la variable Curso, se producen interacciones únicamente en tres variables del Clima de Aula: Implicación, Tareas y Organización (Tabla 4). Respecto a la Implicación ( $p<.01)$. nos encontramos con una misma puntuación del grupo experimental y control en Primero de ESO, sin embargo, en 
Tercero, hay una significativa mayor puntuación tanto respecto al Tercero de ESO del grupo control, como el Primero de ESO del grupo experimental. Por otra parte, la puntuación del Tercer curso del grupo control es significativamente inferior a la del Primer curso del mismo grupo. Por lo tanto, podemos concluir que el programa, respecto a la variable Implicación, únicamente ha sido eficaz en el curso de Tercero de ESO. Esta misma tendencia podemos observarla en la variable Tareas $(p<.001)$ y Organización $(p<.01)$.

Tabla 4. Interacción entre la Condición Experimental y el Sexo en Tercero de ESO

\begin{tabular}{|c|c|c|c|c|c|c|c|}
\hline & Grupo & Sexo & $N$ & Media & D.T. & $F$ & Sig. \\
\hline \multirow{4}{*}{ Implicación } & \multirow{2}{*}{ Experimental } & Mujer & 73 & 4.40 & 3.235 & \multirow{4}{*}{$7.857 * *$} & \multirow{4}{*}{.005} \\
\hline & & Varón & 63 & 5.25 & 2.918 & & \\
\hline & \multirow{2}{*}{ Control } & Mujer & 64 & 4.20 & 2.846 & & \\
\hline & & Varón & 52 & 3.04 & 2.029 & & \\
\hline \multirow{4}{*}{ Ayuda } & \multirow{2}{*}{ Experimental } & Mujer & 73 & 6.07 & 2.854 & \multirow{4}{*}{$5.350^{*}$} & \multirow{4}{*}{.022} \\
\hline & & Varón & 63 & 6.89 & 2.308 & & \\
\hline & \multirow{2}{*}{ Control } & Mujer & 64 & 5.47 & 2.731 & & \\
\hline & & Varón & 52 & 4.77 & 2.323 & & \\
\hline \multirow{4}{*}{ Organización } & \multirow{2}{*}{ Experimental } & Mujer & 73 & 5.14 & 3.533 & \multirow{4}{*}{$7.048 * *$} & \multirow{4}{*}{.008} \\
\hline & & Varón & 62 & 6.21 & 3.084 & & \\
\hline & \multirow{2}{*}{ Control } & Mujer & 64 & 4.63 & 2.820 & & \\
\hline & & Varón & 52 & 3.63 & 2.528 & & \\
\hline
\end{tabular}

$* \mathrm{p}<.05 ; * * \mathrm{p}<.01 . * * * \mathrm{p}<.001$

Tabla 5. Interacción entre la Condición Experimental y el Curso

\begin{tabular}{|c|c|c|c|c|c|c|c|}
\hline & Grupo & Sexo & $N$ & Media & D.T. & $F$ & Sig. \\
\hline \multirow{4}{*}{ Implicación } & \multirow{2}{*}{ Experimental } & $1^{\circ} \mathrm{ESO}$ & 143 & 4.24 & 2.470 & \multirow{4}{*}{$9.220 * *$} & \multirow{4}{*}{.003} \\
\hline & & $3^{\circ} \mathrm{ESO}$ & 136 & 4.79 & 3.111 & & \\
\hline & \multirow{2}{*}{ Control } & $1^{\circ} \mathrm{ESO}$ & 119 & 4.57 & 2.476 & & \\
\hline & & $3^{\circ} \mathrm{ESO}$ & 116 & 3.68 & 2.569 & & \\
\hline \multirow{4}{*}{ Tareas } & \multirow{2}{*}{ Experimental } & $1^{\circ} \mathrm{ESO}$ & 136 & 5.58 & 1.438 & \multirow{4}{*}{$17.327 * * *$} & \multirow{4}{*}{.000} \\
\hline & & $3^{\circ} \mathrm{ESO}$ & 135 & 5.93 & 1.817 & & \\
\hline & \multirow{2}{*}{ Control } & $1^{\circ} \mathrm{ESO}$ & 118 & 5.71 & 1.845 & & \\
\hline & & $3^{\circ} \mathrm{ESO}$ & 116 & 4.83 & 1.528 & & \\
\hline \multirow{4}{*}{ Organización } & \multirow{2}{*}{ Experimental } & $1^{\circ} \mathrm{ESO}$ & 141 & 4.57 & 2.655 & \multirow{4}{*}{$11.170^{* *}$} & \multirow{4}{*}{.001} \\
\hline & & $3^{\circ} \mathrm{ESO}$ & 135 & 5.63 & 3.365 & & \\
\hline & \multirow{2}{*}{ Control } & $1^{\circ} \mathrm{ESO}$ & 119 & 4.82 & 2.577 & & \\
\hline & & $3^{\circ} \mathrm{ESO}$ & 116 & 4.18 & 2.727 & & \\
\hline
\end{tabular}

\section{DISCUSION}

Todos somos conscientes de la importancia de la influencia del profesorado en la motivación de su alumnado. En este sentido, nuestro trabajo, ahonda en la mejora de las habilidades docentes, con el objetivo de mejorar otras variables, tanto internas como externas, que condicionan la motivación, una de ellas, es el clima que se genera en el aula.

Hemos podido comprobar como nuestro programa tiene una mayor eficacia en cursos más superiores dado que en Tercero de ESO mejoran todas las variables medidas referentes al Clima de Aula, mientras que en Primero de ESO únicamente la Competitividad e Innovación, quizás por ser su cambio más fácilmente perceptible por el alumnado respecto a otras, como por ejemplo, las Tareas, en donde el margen de 
cambio es menor debido a los hábitos instaurados en Educación Primaria, y que hacen que las tareas sean más inflexibles y generales que en Tercero de la ESO, con contenidos y asignaturas más diversificadas. Algo parecido ocurre con la Organización y Ayuda.

En cuanto a las diferencias en función del sexo, hemos visto como hay una homogeneidad en Primero de ESO, en donde no existen diferencias, frente a Tercero, en donde se producen diferencias en Autoeficacia para el rendimiento, con una muy superior puntuación de las mujeres del grupo experimental respecto al control frente a puntuaciones similares en los varones, por lo que el programa ha sido diferencialmente más eficaz en las mujeres respecto a los hombres, justo al contrario que en Implicación, con una muy superior puntuación de los varones del grupo experimental respecto al control frente a puntuaciones similares en las mujeres. En el caso de la variable Ayuda y Organización se produce una muy superior puntuación de los varones del grupo experimental respecto al control, justo lo contrario de lo que ocurre con las mujeres, con una muy superior puntuación de las del grupo control respecto al experimental.

Por último, respecto al curso, se produce la misma tendencia en las variables Implicación, Tareas y Organización, con una misma puntuación del grupo experimental y control en Primero de ESO, Sin embargo, en Tercero de ESO hay una significativa mayor puntuación tanto respecto al Tercero de ESO del grupo control, como el Primero de ESO del grupo experimental. Por otra parte, la puntuación del Tercer curso del grupo control es significativamente inferior a la del Primer curso del mismo grupo. En definitiva, el programa únicamente ha sido eficaz en el curso de Tercero de ESO, en donde parece que el profesorado está más abierto a introducir modificaciones y el alumnado más preparado para percibirlas.

\section{Agradecimientos}

Esta aportación forma parte del proyecto de investigación I+D+I SEJ200606978. financiado por el MEC.

\section{REFERENCIAS}

Alonso-Tapia, J. (2005). Motivar en la escuela, motivar en la familia. Madrid: Ediciones Morata. ANECA. (2004). La educación de las titulaciones de maestro al Espacio Europeo de Educación Superior.

Http://www.ua.es/centros/educacion/BORRADOR_DEL_INFORME_FINAL.pdf

Angulo, F. (1999). Entrenamiento y coaching: los peligros de una vía revitalizada. En A. Perez; J. Barquín y J.F. Angulo (Eds.) Desarrollo profesional del docente: Política, Investigación y Práctica. Tres Cantos (Madrid): Akal.

Beltrán, J., García-Alcañiz, E., Moraleda, M.G., Calleja, F. y Santiuste, V. (1987). Psicología de la Educación. Madrid: Eudema.

Benito, A. (2009). La pedagogía no tiene la culpa: un análisis de los problemas de la educación en España. Revista de Educación, 348, 489-501.

Bennet, N. (1979). Estilos de enseñanza y progreso de los alumnos. Madrid: Morata.

Brophy, J. y Good, T.L. (1996). Psicología educativa contemporánea. México: McGraw Hill Interamericana. 
Carrasco, C. y Trianes, M.V. (2010). Clima social, prosocialidad y violencia como predictores de inadaptación escolar en primaria. European Journal of Education and Psychology, 3(2), 229-242.

Carbonero, M.A., Martín-Antón, L.J. y Feijoó, M. (2010). Las creencias irracionales en relación con ciertas conductas de consumo en adolescentes. European Journal of Education and Psychology, 3(2), 287-298.

Fernández-García, J.A. (2008). Modelo explicativo del rendimiento en secundaria: variables cognitivas del profesor y cognitivas, afectivas, motivacionales y escolares de los alumnos. Departamento de Psicología. Universidad de Valladolid. Tesis doctoral.

Flanders, N.A. (1985). Análisis de la interacción didáctica. Madrid: Anaya.

Gage, N.L. (1972). Teacher effectiveness and teacher education: The search of a scientific basses. Palo Alto (California): Pacific Books.

García-Llamas, J.L. (1999). Formación del profesorado: necesidades y demandas. Monografías escuela española. Barcelona: Praxis.

Gregorc, A.F. (1985). Inside Styles: Beyond the basics. Maynard. Mass: Gabriel Systems.

Hervás, R.M. (2005). Estilos de enseñanza y aprendizaje en escenarios educativos. Murcia: Grupo Editorial Universitario.

Lozano-Rodríguez, A. (2005). Estilos de aprendizaje y enseñanza. México: Trillas.

Marchesi, A. (2007). Sobre el bienestar de los docentes: Competencias, emociones y valores. Madrid: Alianza.

Martínez, P. (2007). Aprender y enseñar: Los estilos de aprendizaje y de enseñanza desde la práctica de aula. I.C.E. Universidad de Deusto: Mensajero. SAU.

Perrenaud, P. (2004). Diez nuevas competencias para enseñar. Barcelona: Grao.

Prieto, M.D. y Hervás, R. (1992). El aprendizaje estratégico en las CCSS. Valencia: Cossío.

Román, J.M. (2006). Estrategias docentes. Didáctica de la Educación Física: Tándem 20, 7-22.

Sternberg, R.J. (1990). Más allá del cociente intelectual. Bilbao: DDB

Valle, A., González-Cabanach, R. y Rodríguez, S. (2006). Reflecting on motivation and learning in the new Spanish education act (LOE): talking vs. doing. Papeles del Psicólogo, 27(3), 135-138.

Zabalza, M.A. (2003). Competencias docentes del profesorado universitario: Calidad y desarrollo profesional. Madrid: Narcea.

Woolfolk, A.E. (2006). Psicología Educativa. México: Prentice-Hall Hispanoamericana.

Recibido: 1 de marzo de 2010

Recepción Modificaciones: 10 de noviembre de 2010

Aceptado: 24 de noviembre de 2010 